\title{
Oluwatosin O. Dada wins ABC best paper award
}

\author{
Andrea Pfeifer
}

Published online: 26 June 2011

(C) Springer-Verlag 2011

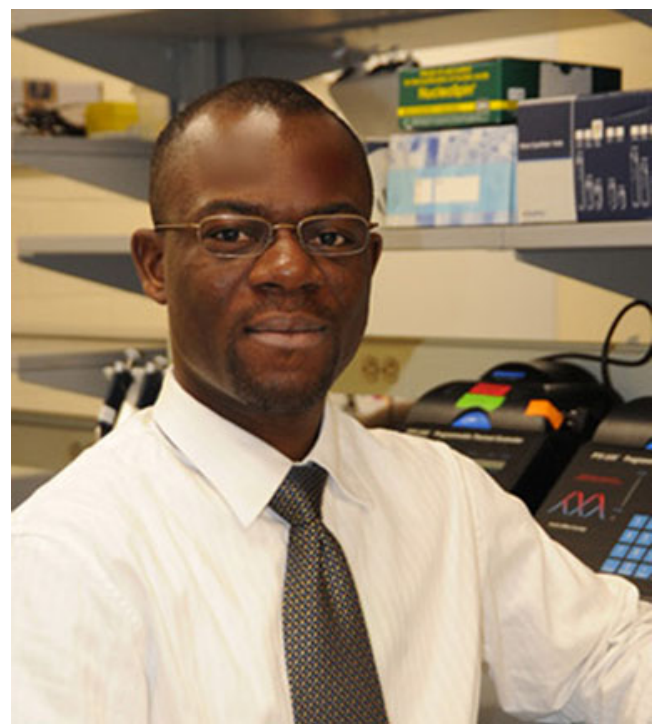

Oluwatosin O. Dada received his Bachelor degree in Industrial Chemistry in 2001 from Olabisi Onabanjo University, Nigeria. He moved to the United States in 2004, where he enrolled in the graduate chemistry program at Utah State University under the direction of Professor Stephen Bialkowski. He received his Ph.D. in 2008 in Analytical Chemistry and spent two years at the University of Washington (Seattle) as a postdoctoral research associate in Professor Norman Dovichi's laboratory, working on ultrasensitive capillary electrophoresis instrumentation for bioanalysis. He currently holds a research assistant professor position at the University of Notre Dame (Indiana), USA, where he continues his work on capillary electrophoresis with laser-induced fluorescence and photothermal instrumentation for bioanalysis.

\footnotetext{
A. Pfeifer $(\square)$

Analytical and Bioanalytical Chemistry, Springer,

Tiergartenstr. 17,

69121 Heidelberg, Germany

e-mail: abc@springer.com
}

This year's Best Paper Award for outstanding work published in Analytical and Bioanalytical Chemistry (ABC) goes to Oluwatosin O. Dada (34), who is the lead author of the paper "Capillary array isoelectric focusing with laser-induced fluorescence detection". Dr Dada's significant contribution to this technology is the development of a state-of-the-art tool for highthroughput capillary isoelectric focusing. The performance of this technology is stunning: it provides the highest throughput isoelectric focusing analysis ever reported, the highest sensitivity ever reported for a high-throughput instrument, and the highest resolution separation ever reported for capillary isoelectric focusing. There is great potential for a wide range of applications of this technology, including characterization of recombinant and therapeutic proteins, diagnosis of disease, and study of systems biology. Accompanied by EUR 1,000, the Award is sponsored by Springer to acknowledge the work of an exceptional young scientist. The article "Capillary array isoelectric focusing with laser-induced fluorescence detection" by Oluwatosin O. Dada, Lauren M. Ramsay, Jane A. Dickerson, Nathan Cermak, Rong Jiang, Cuiru Zhu, and Norman J. Dovichi was published as Paper in Forefront in the $\mathrm{ABC}$ issue entitled "Analytical Tools for Cell Research" (Vol. 397/8, pp. 3305-3310), now freely available online on SpringerLink at http://www.springerlink.com/content/ 681x271158435h58/.

\section{Who is Oluwatosin O. Dada?}

Oluwatosin O. Dada talks about his research, his motivations, and about himself to Andrea Pfeifer. 
What was your motivation to perform research into high-throughput capillary isoelectric focusing?

Capillary electrophoresis was important to the completion of the Genome Project. The motivation for investigating high-throughput capillary isoelectric focusing is its envisaged role in proteomics. Capillary isoelectric focusing can enable much faster separation than classic gel-based methods and is much easier to automate for highthroughput analysis. The instrument provides unprecedented throughput, extraordinary sensitivity, and the most astonishing resolution ever reported for isoelectric focusing separation. The technology will find wide application, including characterization of recombinant and therapeutic proteins, diagnosis of disease, and study of systems biology.

\section{How would you explain your research to your child?}

I would tell my child that I study physical and chemical processes and build instruments that can help to solve some of the problems associated with these processes. For example, I develop tools with the purpose of making it easier for doctors to treat some serious diseases.

What's the trickiest problem you've had to overcome in the research? How did you solve it?

The trickiest problem was setting up the instrument: inserting arrays of several capillaries of decreasing width in a rectangular cuvette of decreasing width and trying to make them behave in a similar manner under an applied electric field. It required dedication, patience, and accuracy.

Where do you see this field heading and how do you see it affecting bioanalytical research?

Analytical spectrochemical techniques have been important in bioanalytical research and continue to produce top-tier bioanalytical tools. I envisage the field forming the major component of micro total-analysis systems, which might completely change the way we conduct bioanalysis.

\section{Which incident/discovery has proved most valuable for your own research?}

Work by Townes, Basov, and Prokhorov on lasers has proved very valuable for my research. Laser technology has trans- formed the way many spectrochemical analyses are performed in a variety of applications. In particular, the pioneering work of Professor Richard N. Zare on laser-excitation detection for electrokinetic separation is very valuable.

\section{Which incident/discovery most inspired you during} your education and scientific career?

Completion of the Human Genome Project is very fascinating. I am personally impressed by the way it globalized biomedical research.

Which recent discovery might prove most valuable to (bio) analytical research or beyond?

Lab-on-a-chip comes to mind, although there are still many challenges to be addressed. It has potential for simplified and inexpensive clinical assays. I can see the technology removing some barriers, enabling good healthcare to be provided in developing countries.

\section{What was the best/worst advice you've ever received?}

All advice, no matter how harsh, has some element of wisdom. The best advice I have received is to "never underestimate your potential".

Whom do you admire the most or who inspired you the most and why?

The support I get from my advisors is very admirable. My colleagues in the laboratory are also inspiring. Without their cooperation, my work would be difficult.

\section{What are your future plans?}

I would like to spend more time using my talent in analytical instrumentation to develop useful tools for solving various health and environmental problems. I look forward to challenging opportunities that will result in this kind of outstanding scientific research.

\section{What do you do in your spare time?}

I spend time with my family and play guitar. 